\title{
Identification of the Key Genes and Pathways in Esophageal Carcinoma
}

\author{
Peng Su, Shiwang Wen, Yuefeng Zhang, Yong Li, Yanzhao Xu, Yonggang Zhu, Huilai Lv, \\ Fan Zhang, Mingbo Wang, and Ziqiang Tian
}

Department of Thoracic Surgery, The Fourth Hospital of Hebei Medical University, Shijiazhuang 050011, China

Correspondence should be addressed to Ziqiang Tian; tizq12@vip.163.com

Received 28 March 2016; Revised 1 July 2016; Accepted 11 July 2016

Academic Editor: Robert Odze

Copyright (c) 2016 Peng Su et al. This is an open access article distributed under the Creative Commons Attribution License, which permits unrestricted use, distribution, and reproduction in any medium, provided the original work is properly cited.

\begin{abstract}
Objective. Esophageal carcinoma (EC) is a frequently common malignancy of gastrointestinal cancer in the world. This study aims to screen key genes and pathways in EC and elucidate the mechanism of it. Methods. 5 microarray datasets of EC were downloaded from Gene Expression Omnibus. Differentially expressed genes (DEGs) were screened by bioinformatics analysis. Gene Ontology (GO) enrichment, Kyoto Encyclopedia of Genes and Genomes (KEGG) enrichment, and protein-protein interaction (PPI) network construction were performed to obtain the biological roles of DEGs in EC. Quantitative real-time polymerase chain reaction (qRTPCR) was used to verify the expression level of DEGs in EC. Results. A total of 1955 genes were filtered as DEGs in EC. The upregulated genes were significantly enriched in cell cycle and the downregulated genes significantly enriched in Endocytosis. PPI network displayed CDK4 and CCT3 were hub proteins in the network. The expression level of 8 dysregulated DEGs including CDK4, CCT3, THSD4, SIM2, MYBL2, CENPF, CDCA3, and CDKN3 was validated in EC compared to adjacent nontumor tissues and the results were matched with the microarray analysis. Conclusion. The significantly DEGs including CDK4, CCT3, THSD4, and SIM2 may play key roles in tumorigenesis and development of EC involved in cell cycle and Endocytosis.
\end{abstract}

\section{Introduction}

Esophageal carcinoma (EC) is the sixth leading cause of cancer mortality in males and the ninth leading cause of cancer mortality in females in 2012 worldwide [1]. The highest incident rates of EC are found in Eastern Asia, Southern Africa, and Eastern Africa and the lowest incidence rate of EC is found in Western Africa [1]. Esophageal carcinoma is usually 3 to 4 times more common among men than women. The 5-year overall survival ranges from $15 \%$ to $25 \%$ [2]. In China, it is predicted that EC is the fourth leading cause of cancer deaths in males and females after lung and bronchus, stomach, and liver in 2015 [3].

EC is classified as esophageal squamous cell carcinoma (ESCC) and esophageal adenocarcinoma (EAC) according to histological type and ESCC is the predominant histological type of EC in the world [2]. It is reported that tobacco consumption, alcohol consumption, and low intake of fruits and vegetables are major risk factors for ESCC [4]. Overweight, obesity, gastroesophagus reflux disease (GERD), and Barrett's esophagus increase incidence risk of EAC $[1,5]$.

In addition to the above-mentioned environmental factors, abnormal expression of miRNA and genes and methylation of genes and SNPs are associated with EC tumorigenesis and development. miR-219-1 rs107822G > A polymorphism might significantly decrease ESCC risk through changing individual susceptibility to Chinese Kazakhs [5]. The cases carrying the GG variant homozygote have a significant 2.81fold increased risk of EC [6]. miR-330-3p promotes cell growth, cell migration, and invasion and inhibits cisplatininduced apoptosis in ESCC cells via suppression of PDCD4 expression [7]. miR-199a-5p downregulation contributes to enhancing EC cell proliferation through upregulation of mitogen-activated protein kinase kinase kinase-11 [8]. DACT2 is frequently methylated in human esophageal cancer; methylated DATC2 accelerates esophageal cancer development by activating Wnt signaling [9]. RUNX3 methylation 
is associated with an increased risk, progression, and poor survival in EC [10].

Currently, the molecular mechanism of EC was unclear. In this study, we used bioinformatics methods to analyze the mRNA expression data of EC, which were available on the GEO database, to identify key genes and pathways in EC, aiming to provide valuable information for further pathogenesis mechanism elucidation and provide ground work for therapeutic targets identification for EC.

\section{Materials and Methods}

2.1. Expression Profile Microarray. Gene expression profiles data were downloaded from the Gene Expression Omnibus (GEO) data repository (http://www.ncbi.nlm.nih.gov/geo/). The datasets of patients receiving preoperative treatment before oesophagectomy and cell lines receiving drug stimulus were excluded. Total of 5 mRNA expression datasets of EC tissues/cell lines comprising GSE53625, GSE33810, GSE17351, GSE9982, and GSE12737 were included in our study.

2.2. Identification of DEGs. The raw data of the mRNA expression profiles were downloaded and analyzed by $\mathrm{R}$ language software [11]. Background correction, quartile data normalization, and probe summarization were applied for the original data. The limma [12] method in Bioconductor (http://www.bioconductor.org/) was used to identify genes which were differentially expressed between EC and normal controls; the significance of DEGs was calculated by $t$-test and was represented by $p$ value. To reduce the risk of false positives, $p$ values were adjusted for multiple testing using the Benjamini-Hochberg False Discovery Rate (FDR) method. The corrected $p$ value was represented by FDR [13]. FDR < 0.05 were considered as the cutoff values for DEG screening.

2.3. Gene Ontology Analysis. GO is a useful tool for collecting a large number of gene annotation terms [14]. The Database for Annotation, Visualization, and Integrated Discovery (DAVID) [15], is bioinformatics resources consisting of an integrated biological knowledgebase and analytic tools aimed at systematically extracting biological functional annotation from large gene/protein lists, such as being derived from high-throughput genomic experiments. To gain the in-depth understanding of the biological functions of DEGs, DAVID tool was used to obtain the enriched GO terms of DEGs based on the hypergeometric distribution to compute $p$ values, which were corrected by the Benjamini and Hochberg FDR method for multiple hypothesis testing. FDR $<0.05$ was set as the threshold value.

2.4. KEGG Enrichment Pathways. KEGG is a database resource for understanding functions of genes list from molecular level [16]. GeneCoDis3 is a valuable tool to functionally interpret results from experimental techniques in genomics [17]. This web-based application integrates different sources of information for finding groups of genes with similar biological meaning. The enrichment analysis of GeneCoDis3 is essential in the interpretation of high-throughput experiments. In the study, GeneCoDis3 software was used to test the statistical enrichment of DEGs in KEGG pathways. $p<0.05$ was set as the threshold value.

2.5. PPI Interaction Network. The Biological General Repository for Interaction Datasets (BioGRID: http://thebiogrid .org/) is an open access archive of genetic and protein interactions that are curated from the primary biomedical literature for all major model organism species including budding yeast Saccharomyces cerevisiae, the fission yeast Schizosaccharomyces pombe, and the model plant Arabidopsis thaliana. In a word, BioGRID is a depository for genetic and protein interactions based on experimental verification [18]. The top 10 upregulated genes and top 10 downregulated genes between EC and normal controls were subjected to BioGRID database to get the predicted PPIs of these DEGs. The PPIs were visualized in Cytoscape [17].

2.6. qRT-PCR Validation. Total RNA of fresh paired EC tumor and adjacent nontumor specimens were extracted using TRIzol reagent (Invitrogen, CA, USA). The SuperScript III Reverse Transcription Kit (Invitrogen, CA, USA) was used to synthesize the cDNA. qRT-PCR reactions were performed using Power SYBR Green PCR Master Mix (Applied Biosystems, Foster City, CA) on the Applied Biosystems 7500 (Foster City, CA, USA). $\beta$-actin was used as internal control for mRNA detected. The relative expression of genes was calculated using the comparative Ct methods [19]. The PCR primers were used as shown in supplementary Table S3 in Supplementary Material available online at http://dx.doi.org/10.1155/2016/2968106.

\section{Results}

3.1. Identification of DEGs. Five mRNA expression profiles including 208 EC samples and 195 normal controls were downloaded and analyzed, as shown in Table 1. 208 EC samples comprised 207 squamous cell carcinoma samples and 1 adenocarcinoma sample. 1955 DEGs were identified in EC compared to normal control, including 919 upregulated and 1036 downregulated genes. The top 10 significantly upregulated and downregulated genes were listed in Table 2. The most significantly up- and downregulated genes were CDK4 and THSD4, respectively. The full list of DEGs in EC was shown in supplementary Table S1.

3.2. GO Analysis of DEGs. Following GO analyses for upand downregulated DEGs, significant GO terms including biological process, cellular component, and molecular function were collected. For upregulated DEGs, cell cycle was the most significant enrichment of biological process; membrane-enclosed lumen was the highest enrichment of cellular component; nucleotide binding was the highest enrichment of molecular function, as shown in Table 3. For downregulated DEGs, response to wounding was the most significant enrichment of biological process; actin cytoskeleton was the highest enrichment of cellular component and 
TABLE 1: The information of gene expression microarrays of EC.

\begin{tabular}{|c|c|c|c|c|c|c|}
\hline GEO ID & Platform & Case: control & Sample type & Country & Time & Author \\
\hline GSE53625 & $\begin{array}{c}\text { GPL18109 CBC Homo } \\
\text { sapiens lncRNA + mRNA } \\
\text { microarray V2.0 }\end{array}$ & $179: 179$ & $\begin{array}{l}\text { Esophageal squamous } \\
\text { cell carcinoma }\end{array}$ & China & 2014 & Li et al. [42] \\
\hline GSE33810 & $\begin{array}{c}\text { GPL570 [HG-U133_Plus_2] } \\
\text { Affymetrix Human } \\
\text { Genome U133 Plus } 2.0 \\
\text { Array }\end{array}$ & $2: 1$ & $\begin{array}{l}\text { Esophageal squamous } \\
\text { cell carcinoma }\end{array}$ & HK & 2013 & Chen et al. [43] \\
\hline GSE17351 & $\begin{array}{c}\text { GPL570 [HG-U133_Plus_2] } \\
\text { Affymetrix Human } \\
\text { Genome U133 Plus } 2.0 \\
\text { Array }\end{array}$ & $5: 5$ & $\begin{array}{l}\text { Esophageal squamous } \\
\text { cell carcinoma }\end{array}$ & USA & 2009 & Long et al. [44] \\
\hline GSE9982 & $\begin{array}{c}\text { GPL1928 CodeLink Human } \\
\text { 20K ver4.1 }\end{array}$ & $20: 2$ & $\begin{array}{l}\text { Esophageal squamous } \\
\text { cancer }\end{array}$ & Japan & 2006 & Shimokuni et al. [45] \\
\hline GSE12737 & $\begin{array}{c}\text { GPL7262 Human } \\
\text { ORESTES NoMatch 4.8k } \\
\text { v1.0 }\end{array}$ & $2: 8$ & $\begin{array}{l}\text { Squamous cell \& } \\
\text { adenocarcinoma }\end{array}$ & Brazil & 2009 & Mello et al. [46] \\
\hline
\end{tabular}

EC: esophageal carcinoma.

TABLE 2: The top 10 up-regulated and top 10 down-regulated DEGs in EC.

\begin{tabular}{|c|c|c|c|}
\hline Gene ID & Gene symbol & Official full name & FDR \\
\hline \multicolumn{4}{|c|}{ Upregulated (top 10) } \\
\hline 1019 & CDK4 & Cyclin-dependent kinase 4 & 0.0002252 \\
\hline 4605 & MYBL2 & MYB protooncogene like 2 & 0.0002252 \\
\hline 7203 & ССТ3 & Chaperonin containing TCP1 subunit 3 & 0.0003378 \\
\hline 83461 & CDCA3 & Cell division cycle associated 3 & 0.0004504 \\
\hline 1033 & CDKN3 & Cyclin-dependent kinase inhibitor 3 & 0.0004504 \\
\hline 1063 & CENPF & Centromere protein $\mathrm{F}$ & 0.0004729 \\
\hline 9156 & EXO1 & Exonuclease 1 & 0.0004729 \\
\hline 79075 & DSCC1 & DNA replication and sister chromatid cohesion 1 & 0.0005405 \\
\hline 4751 & NEK2 & NIMA related kinase 2 & 0.0005405 \\
\hline \multicolumn{4}{|c|}{ Downregulated (top 10) } \\
\hline 79875 & THSD4 & Thrombospondin type 1 domain containing 4 & 0.0002252 \\
\hline 79026 & AHNAK & AHNAK nucleoprotein & 0.0004729 \\
\hline 6493 & SIM2 & Single-minded family bHLH transcription factor 2 & 0.0004729 \\
\hline 7881 & KCNAB1 & Potassium voltage-gated channel subfamily A member regulatory beta subunit 1 & 0.0005405 \\
\hline 90865 & IL33 & Interleukin 33 & 0.0008812 \\
\hline 55287 & TMEM40 & Transmembrane protein 40 & 0.0008812 \\
\hline 966 & CD59 & CD59 molecule & 0.0015608 \\
\hline 5121 & PCP4 & Purkinje cell protein 4 & 0.0015608 \\
\hline 22885 & ABLIM3 & Actin binding LIM protein family member 3 & 0.0016629 \\
\hline 3590 & IL11RA & Interleukin 11 receptor subunit alpha & 0.0016629 \\
\hline
\end{tabular}

EC: esophageal carcinoma; FDR: false discovery rate.

cytoskeletal protein binding was the highest enrichment of molecular function, as shown in Table 4.

3.3. KEGG Enrichment Pathways of DEGs. Following KEGG enrichment analysis for DEGs, significant KEGG terms were collected. The pathways enriched by 919 upregulated DEGs were mainly related to cell cycle, RNA transport, and p53 signaling pathway (Table 5). 1036 downregulated DEGs were significantly enriched in Endocytosis, focal adhesion, and vascular smooth muscle contraction, as shown in Table 6.
3.4. PPI Network Construction. Based on data from the BioGRID database, the PPI network was the top 10 upregulated and downregulated DEGs which were constructed by Cytoscape software (Figure 1). The network consisted of 451 nodes and 499 edges. In the PPI networks the nodes with high degree are defined as hub proteins. The most significant hub proteins in the PPI network were CDK4 (degree = 132) and CCT3 (degree $=127$ ); as shown in Figure 1, the red circular nodes represent upregulated DEGs and green circular nodes represent downregulated DEGs, respectively. 
TABLE 3: GO annotation of upregulated DEGs in EC.

\begin{tabular}{|c|c|c|c|c|}
\hline GO ID & GO term & Count & $p$-value & FDR \\
\hline \multicolumn{5}{|c|}{ Biological process } \\
\hline GO:0007049 & Cell cycle & 152 & $4.10 E-13$ & $7.59 E-10$ \\
\hline GO:0022402 & Cell cycle process & 118 & $3.44 E-12$ & $6.36 E-09$ \\
\hline GO:0022403 & Cell cycle phase & 90 & $2.00 E-10$ & $3.71 E-07$ \\
\hline GO:0000278 & Mitotic cell cycle & 82 & $5.34 E-10$ & $9.87 E-07$ \\
\hline GO:0051301 & Cell division & 67 & $8.80 E-09$ & $1.63 E-05$ \\
\hline GO:0000279 & M phase & 70 & $6.27 E-08$ & $1.16 E-04$ \\
\hline GO:0000087 & M phase of mitotic cell cycle & 49 & $3.38 E-06$ & 0.0062547 \\
\hline GO:0000280 & Nuclear division & 48 & $4.65 E-06$ & 0.0086014 \\
\hline GO:0007067 & Mitosis & 48 & $4.65 E-06$ & 0.0086014 \\
\hline GO:0048285 & Organelle fission & 49 & $6.41 E-06$ & 0.011854 \\
\hline GO:0033554 & Cellular response to stress & 95 & $2.02 E-05$ & 0.0373322 \\
\hline \multicolumn{5}{|c|}{ Cellular component } \\
\hline GO:0031974 & Membrane-enclosed lumen & 276 & $1.12 E-10$ & $1.65 E-07$ \\
\hline GO:0043233 & Organelle lumen & 270 & $2.41 E-10$ & $3.56 E-07$ \\
\hline GO:0043232 & Intracellular non-membrane-bounded organelle & 359 & $8.74 E-10$ & $1.29 E-06$ \\
\hline GO:0043228 & Non-membrane-bounded organelle & 359 & $8.74 E-10$ & $1.29 E-06$ \\
\hline GO:0070013 & Intracellular organelle lumen & 259 & $4.54 E-09$ & $6.71 E-06$ \\
\hline GO:0031981 & Nuclear lumen & 216 & $1.90 E-08$ & $2.80 E-05$ \\
\hline GO:0000775 & Chromosome, centromeric region & 36 & $2.52 E-08$ & $3.72 E-05$ \\
\hline GO:0005829 & Cytosol & 192 & $1.36 E-06$ & 0.0020016 \\
\hline GO:0015630 & Microtubule cytoskeleton & 92 & $4.62 E-06$ & 0.0068255 \\
\hline GO:0000793 & Condensed chromosome & 32 & $6.75 E-06$ & 0.009972 \\
\hline GO:0000779 & Condensed chromosome, centromeric region & 21 & $7.55 E-06$ & 0.011151 \\
\hline GO:0044427 & Chromosomal part & 69 & $9.92 E-06$ & 0.0146408 \\
\hline GO:0005635 & Nuclear envelope & 43 & $1.37 E-05$ & 0.0202598 \\
\hline GO:0000777 & Condensed chromosome kinetochore & 19 & $1.48 E-05$ & 0.0219025 \\
\hline GO:0005694 & Chromosome & 78 & $1.75 E-05$ & 0.02589 \\
\hline GO:0000776 & Kinetochore & 22 & $2.72 E-05$ & 0.0401619 \\
\hline \multicolumn{5}{|c|}{ Molecular function } \\
\hline GO:0000166 & Nucleotide binding & 305 & $5.53 E-06$ & 0.0090275 \\
\hline GO:0017076 & Purine nucleotide binding & 266 & $5.55 E-06$ & 0.0090714 \\
\hline GO:0030554 & Adenyl nucleotide binding & 223 & $1.07 E-05$ & 0.0175078 \\
\hline GO:0001883 & Purine nucleoside binding & 225 & $1.49 E-05$ & 0.0242774 \\
\hline GO:0032555 & Purine ribonucleotide binding & 252 & $2.35 E-05$ & 0.0383944 \\
\hline GO:0032553 & Ribonucleotide binding & 252 & $2.35 E-05$ & 0.0383944 \\
\hline GO:0001882 & Nucleoside binding & 225 & $2.44 E-05$ & 0.0398342 \\
\hline
\end{tabular}

EC: esophageal carcinoma; FDR: false discovery rate.

3.5. qRT-PCR Validation of DEGs in EC Tissues. To validate the microarray analysis data, the expression of DEGs including CCT3, CDK4, MYBL2, CENPF, CDKN3, CDCA3, THSD4, and SIM2 was detected by qRT-PCR in 5 paired EC tumor and adjacent nontumor tissues. The 5 patients received surgery treatment in Fourth Hospital of Hebei Medical University. The histological type of 5 subjects was ESCC and the detailed information of subjects was shown in supplementary Table S2. As shown in Figures 2(a) and 2(b) the expression level of CCT3 and MYBL2 was significantly upregulated in ESCC. CDK4, CENPF, CDKN3, and CDCA3 had the upregulation tendency in ESCC (Figures 2(c)-2(f)), respectively. SIM2 was significantly downregulated in ESCC
(Figure 2(g)). THSD4 had the downregulation tendency in ESCC (Figure 2(h)). The qRT-PCR results were matched with the microarray analysis.

\section{Discussion}

CDK4 was identified as the most significantly upregulated gene in our microarray analysis and it had an upregulated tendency in EC tissues through the qRT-PCR validation. CDK4 was the hub protein and interacted with 132 genes in the regulatory network. CDK4 was significantly enriched in cell cycle, measles, small cell lung cancer, and pathways in cancer. CDK4 encodes cyclin-dependent kinase 4 , a member of the 
TABLE 4: GO annotation of downregulated DEGs in EC.

\begin{tabular}{|c|c|c|c|c|}
\hline GO ID & GO term & Count & $p$ value & FDR \\
\hline \multicolumn{5}{|c|}{ Biological process } \\
\hline GO:0009611 & Response to wounding & 65 & $1.98 E-08$ & $3.57 E-05$ \\
\hline GO:0042060 & Wound healing & 33 & $5.75 E-08$ & $1.04 E-04$ \\
\hline GO:0030097 & Hemopoiesis & 32 & $1.85 E-05$ & 0.0334238 \\
\hline GO:0007167 & Enzyme linked receptor protein signaling pathway & 41 & $2.03 E-05$ & 0.0365533 \\
\hline GO:0030036 & Actin cytoskeleton organization & 31 & $2.05 E-05$ & 0.0370181 \\
\hline GO:0048534 & Hemopoietic or lymphoid organ development & 34 & $2.06 E-05$ & 0.0372021 \\
\hline GO:0007155 & Cell adhesion & 69 & $2.10 E-05$ & 0.0378896 \\
\hline GO:0042692 & Muscle cell differentiation & 21 & $2.14 E-05$ & 0.0386651 \\
\hline GO:0022610 & Biological adhesion & 69 & $2.19 E-05$ & 0.0394751 \\
\hline GO:0007178 & Transmembrane receptor protein serine/threonine kinase signaling pathway & 19 & $2.53 E-05$ & 0.0456886 \\
\hline \multicolumn{5}{|c|}{ Cellular component } \\
\hline GO:0015629 & Actin cytoskeleton & 36 & $5.84 E-06$ & 0.008305 \\
\hline GO:0005794 & Golgi apparatus & 83 & $7.36 E-06$ & 0.0104637 \\
\hline GO:0005856 & Cytoskeleton & 118 & $1.23 E-05$ & 0.0175254 \\
\hline \multicolumn{5}{|c|}{ Molecular function } \\
\hline GO:0008092 & Cytoskeletal protein binding & 59 & $8.55 E-07$ & 0.0013403 \\
\hline
\end{tabular}

EC: esophageal carcinoma; FDR: false discovery rate.

Ser/Thr protein kinase family, which plays an important role in cell cycle G1 phase progression and G1/S transition. In our study, CDK1, CDK6, and CDK10 showed upregulation in EC. CDK1, CDK6, and CDK4 were significantly enriched in cell cycle pathway. CDK4 is overexpression in several cancer comprising of breast cancer, pancreas cancer, clear cell renal cell carcinoma, and colorectal cancer [20-23]. Downregulation of MALAT1 (long noncoding RNA metastasis-associated lung adenocarcinoma transcript 1 ) inhibits breast cancer cell proliferation and cell cycle progression in vitro and in vivo through miR-124 downregulation and CDK4 upregulation $[20,24]$. Overexpression of cyclin D1/CDK4 is regulated by CEACAM6 and promotes cell proliferation in human pancreatic carcinoma [21]. CDK4 and CDK6 expression are decreased by miR-1 and contribute to inhibition of cell cycle progression and metastasis in clear cell renal cell carcinoma [22].

CCT3 was the top 3 upregulation DEGs in EC (Table 2). The qRT-PCR displayed that CCT3 was significantly upregulated in EC, which was in accordance with our microarray analysis (Figure 2). CCT3 interacted with 127 genes in the PPI network (Figure 1). CCT3 encodes chaperonin containing TCP1 subunit 3 , a molecular chaperone, which is a member of the chaperonin containing TCP1 complex (CCT). In our study, CCT2, CCT4, CCT5, and CCT7 were upregulated in EC compared to normal controls, respectively. CCT3 depletion suppresses cell proliferation by inducing mitotic arrest at prometaphase and apoptosis eventually in HCC in vitro. Clinically, overexpression of CCT3 predicts poor prognosis in hepatocellular carcinoma patients after hepatectomy $[25,26]$. CCT3 is significantly associated with carboplatin resistance in ovarian cancer patients after surgery treatment [27]. The proteomic-based study shows that patients with cholangiocarcinoma (CCA) which are positive for CCT3 and
CCT3 might be potential biomarker for the diagnosis of CCA [28]. To our knowledge, this is the first report about CCT3 expressed status in EC and the biological function of upregulated CCT3 in EC needs further exploration.

THSD4 was the most downregulated DGE in EC through microarray analysis. The expression level of THSD4 had no significance in EC compared to normal controls but had the downregulated tendency in EC. THSD4 encodes thrombospondin type 1 domain containing 4 . The methylated status of THSD4 shows positive correlation with short survival in glioblastoma patients and hypermethylation of THSD4 indicates poor survival [29]. The expression of THSD4 is regulated by GATA3 and mediates transformation of normal cells into breast cancer through deregulation of THSD4 [30]. The role of downregulated THSD4 in EC is unclear, and the investigation needs to be carried out in the future.

SIM2 was significantly downregulated in EC (Figure 2). SIM2 encodes single-minded family bHLH transcription factor 2. SIM2-s was dysregulated in glioma, prostate cancer, breast cancer, colorectal cancer, and ESCC [31-35]. SIM2s is downregulated in human breast cancer samples and it suppresses tumor activity through decreased expression of matrix metalloprotease-3. In breast cancer, SIM2s is downregulated. It is a key regulator of mammary-ductal development. SIM2s inhibition is associated with cell invasive and EMT-like phenotype through regulating matrix metalloprotease-3 expression [34, 36] It is reported that SIM2s is downregulated in 70\% ESCC tissues, which is consistent with our qRT-PCR verification [35]. SIM2 overexpression results in increase of drug- and radio-sensitivities in ESCC in vivo and in vitro and patients with high expression level of SIM2 are associated with favorable prognosis before chemotherapy [35]. It is suggested that SIM2 plays vital roles in EC onset and progression. 


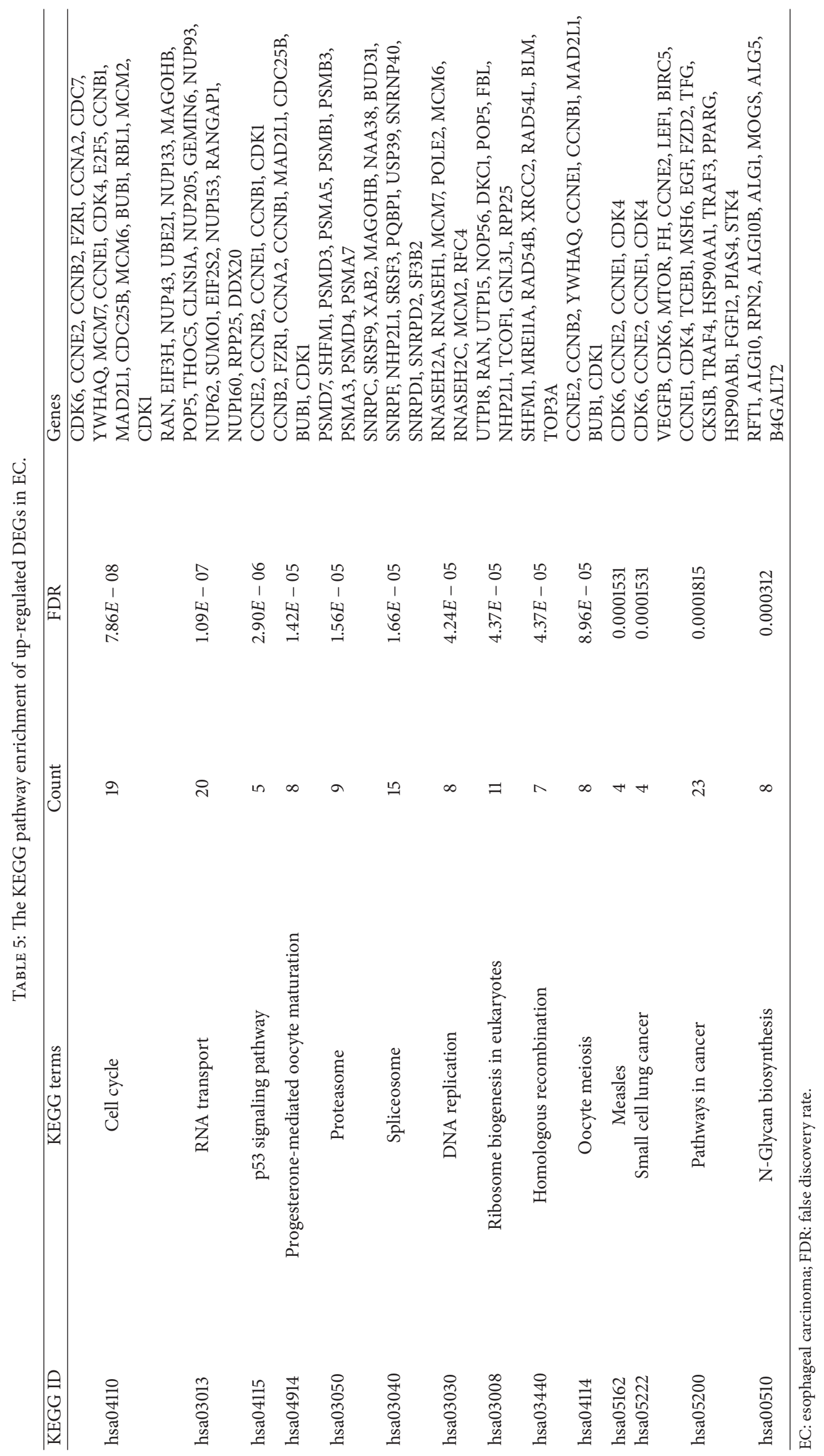




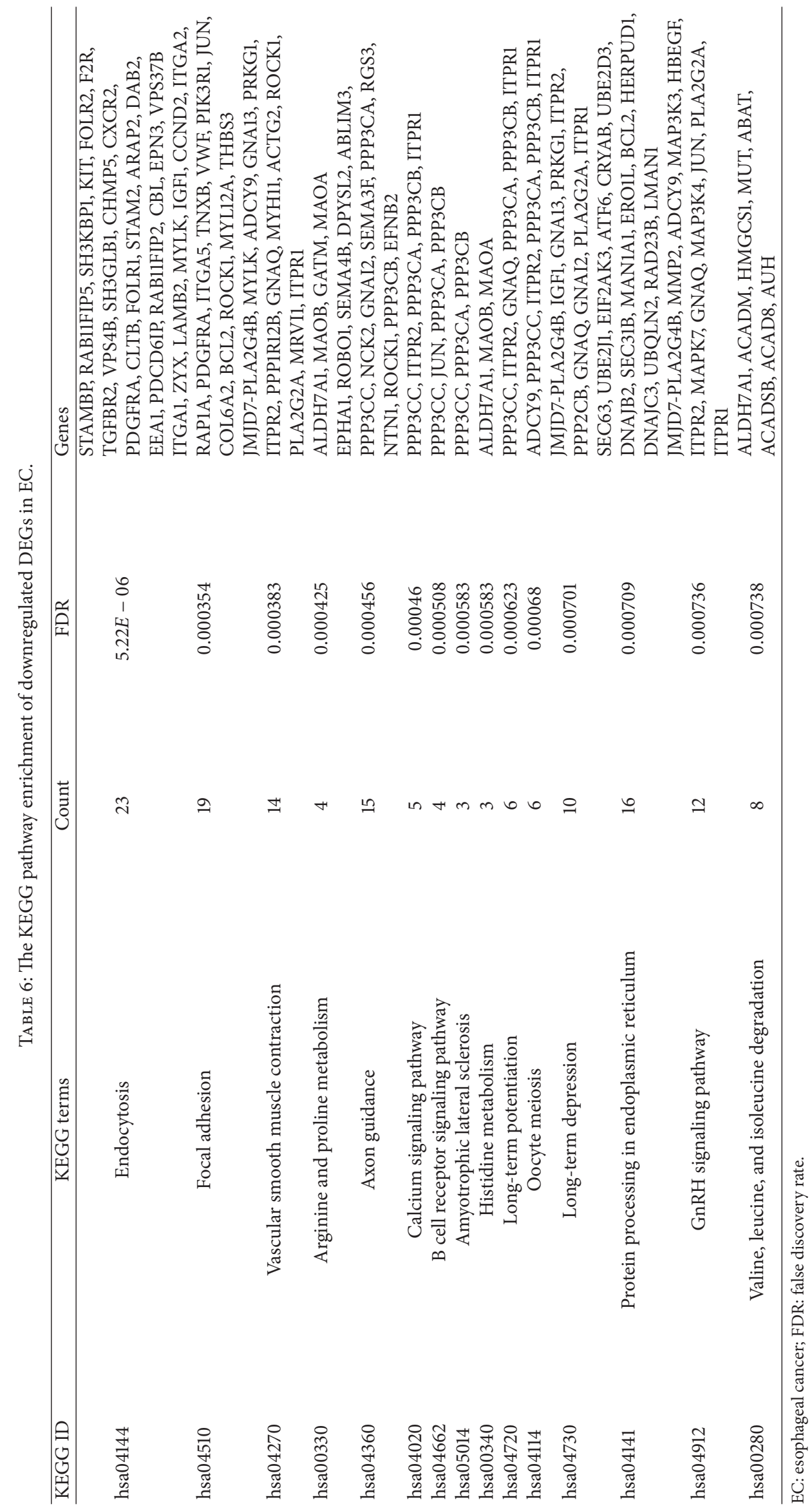




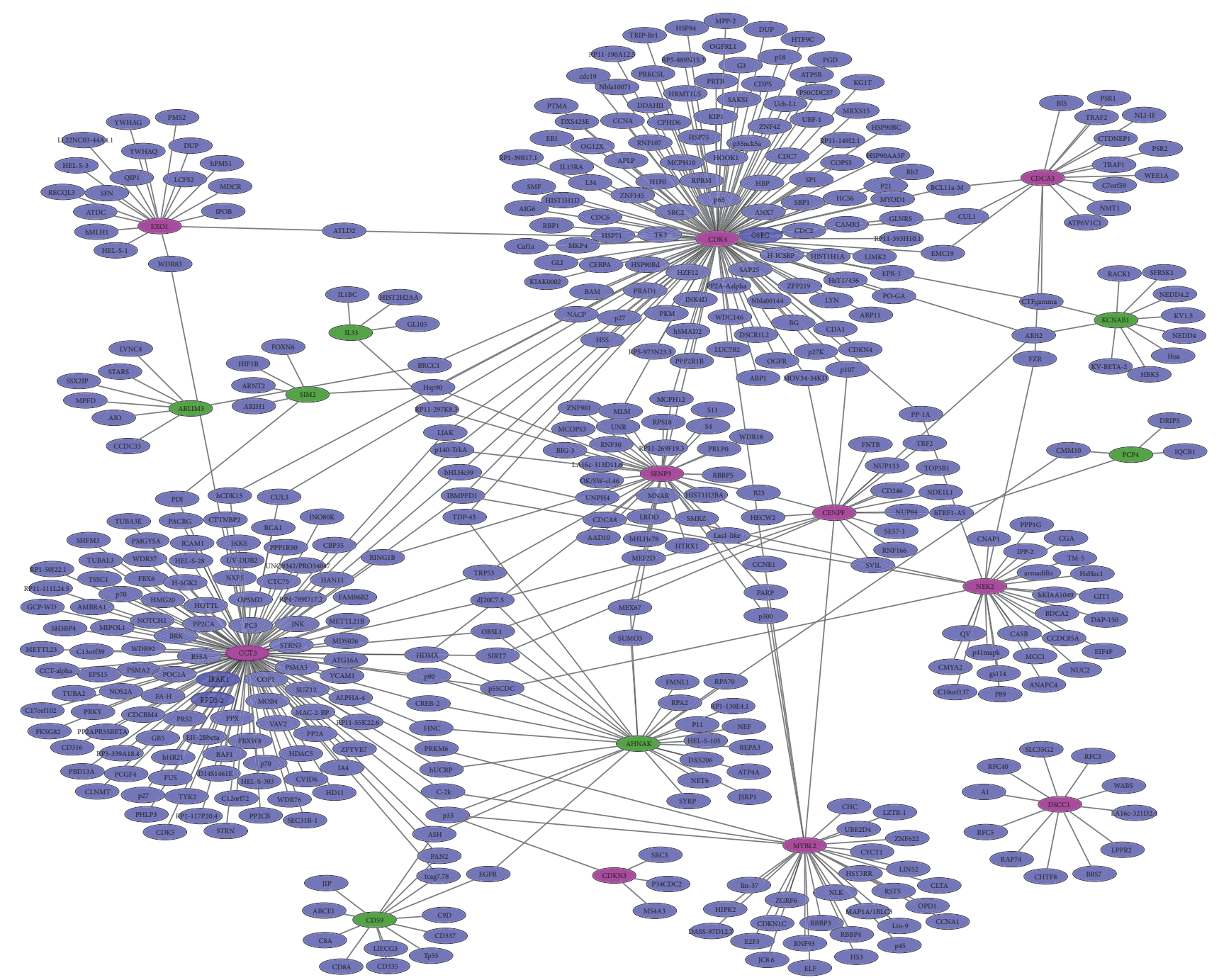

FIGURE 1: The protein-protein network of top 10 up- and downregulated DEGs in EC. The green circular nodes represent downregulation DEGs in EC; the red circular nodes represent downregulation DEGs in EC. Solid lines indicate interaction between DEGs and proteins.

MYBL2, CENPF, CDKN3, and CDCA3 were upregulated in EC tissues (Figure 2). MYBL2 is frequently amplified in gastroesophageal cancer cell lines and Barrett's adenocarcinoma [37, 38]. CENPF is frequently amplified in region around 1q32-q41 and is overexpressed in ESCC cell line [39]. CDKN3 is upregulated in $68.0 \%$ of the epithelial ovarian cancer samples and lung adenocarcinoma patients and is correlated with poor patient survival $[40,41]$. CDCA3 expression status in EC was firstly reported in our study. The molecular mechanism of MYBL2, CENPF, CDKN3, and CDCA3 in EC is needed to be explored.

\section{Conclusions}

We identified 1955 DEGs comprising 919 upregulated genes and 1036 downregulated genes in EC. DEGs including CDK4, CCT3, THSD4, and SIM2 were verified in EC tissues through qRT-PCR. CDK4 and CCT3 were hub proteins in the PPI interaction network. We found that some genes including CDK4, CCT3, THSD4, and SIM2 may play essential roles in EC through cell cycle, RNA transport, Endocytosis, and focal adhesion signaling pathways. The genes could also be considered as potential candidate biomarkers for therapeutic targets for this malignancy. Furthermore, our study would shed light on the molecular mechanism underlying tumorigenesis of EC.

\section{Competing Interests}

All of the authors declare that they have no conflict of interests.

\section{Acknowledgments}

The work was supported by Major Medical Scientific Research Subject of Hebei Province (zd2013044). 


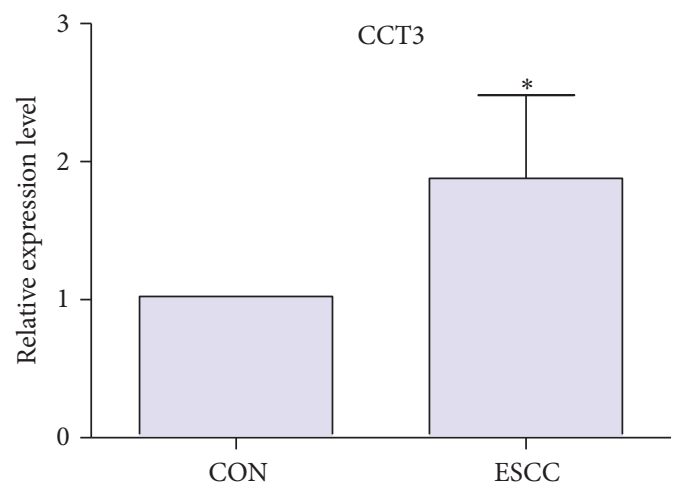

(a)

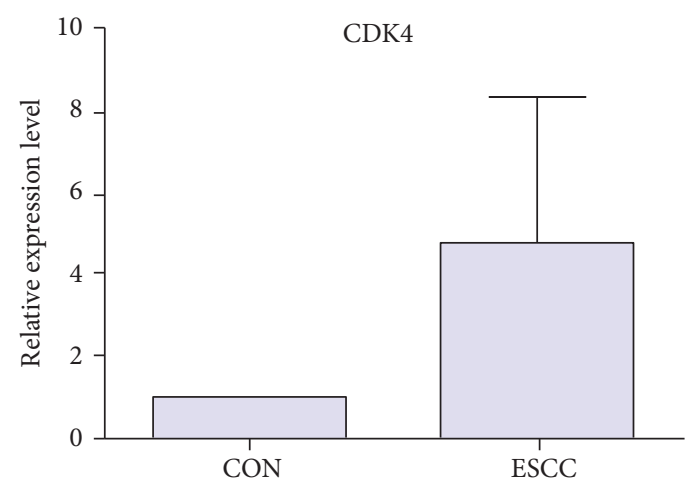

(c)

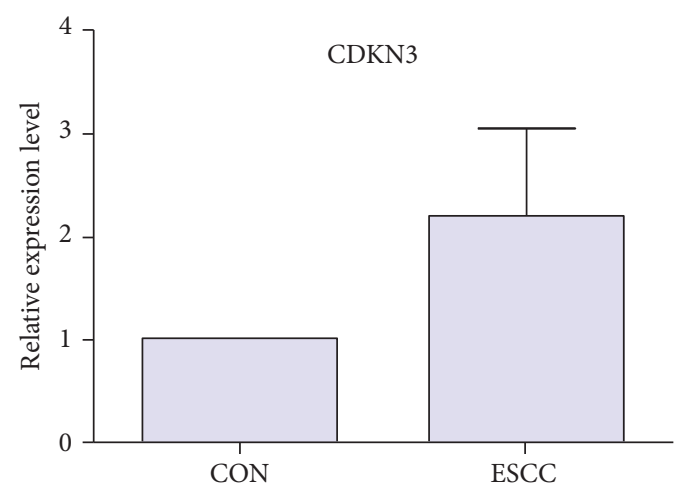

(e)

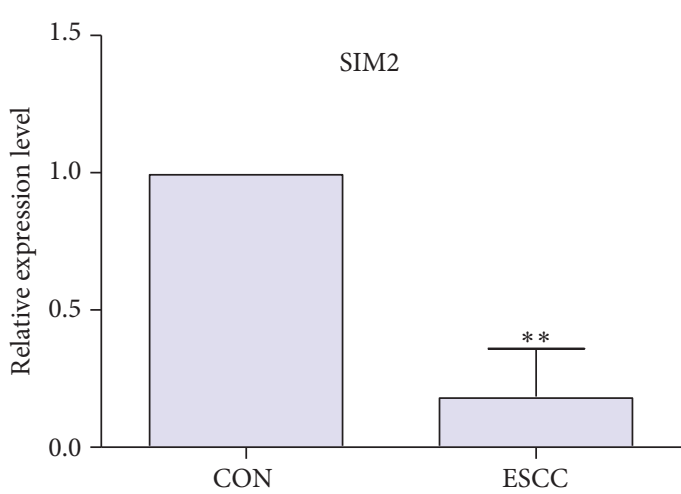

(g)

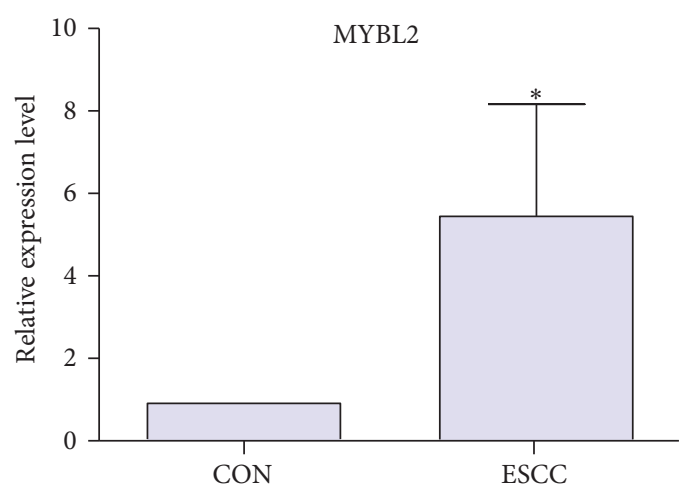

(b)

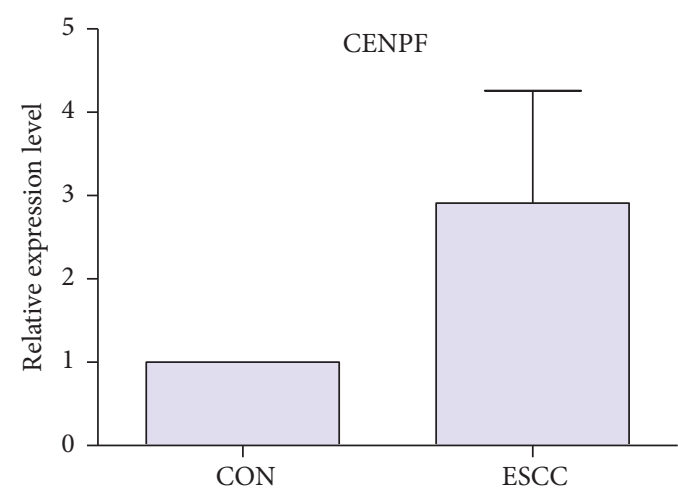

(d)

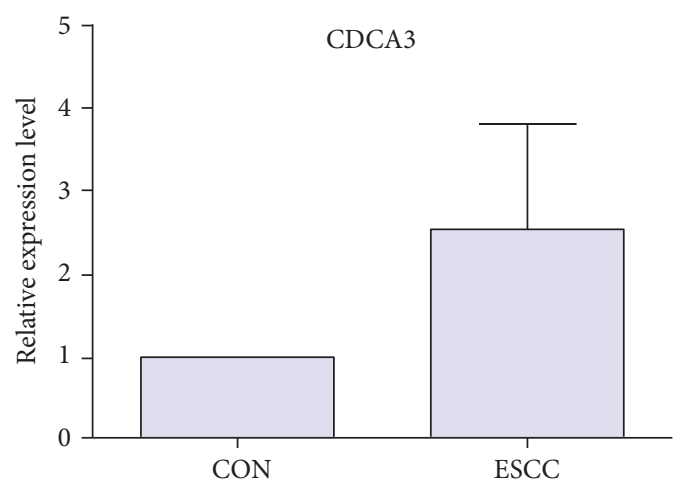

(f)

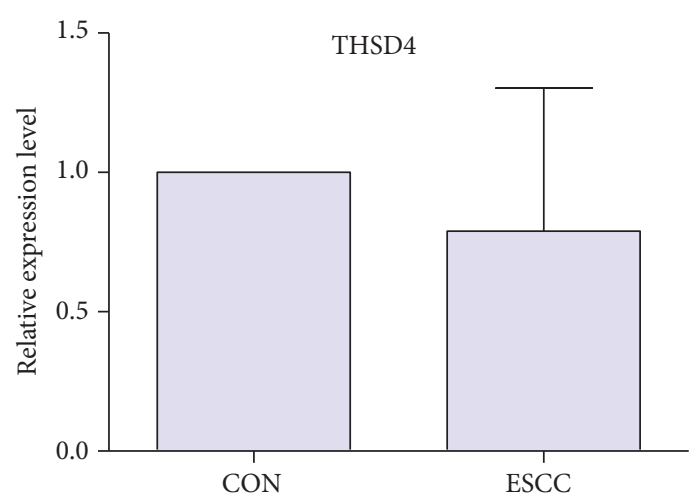

(h)

FIGURE 2: The qRT-PCR validation of the expression level of DEGs in EC compared to adjacent nontumor tissues. (a) CCT3; (b) MYBL2; (c) CDK4; (d) CENPF; (e) CDKN3; (f) CDCA3; (g) SIM2; (h) THSD4. EC: esophageal carcinoma; CON: adjacent nontumor tissues of ESCC. At least three independent experiments were performed for statistical evaluation. qRT-PCR experimental data were expressed as means \pm SD. The statistical significance was evaluated using Student's $t$-test and $p<0.05$ was considered as a significant difference. 


\section{References}

[1] R. Siegel, D. Naishadham, and A. Jemal, "Cancer statistics, 2012," CA: A Cancer Journal for Clinicians, vol. 62, no. 1, pp. 10-29, 2012.

[2] P. C. Enzinger and R. J. Mayer, "Esophageal cancer," The New England Journal of Medicine, vol. 349, no. 23, pp. 2241-2252, 2003.

[3] W. Chen, R. Zheng, P. D. Baade et al., "Cancer statistics in China, 2015," CA: A Cancer Journal for Clinicians, vol. 66, no. 2, pp. 115132, 2016.

[4] M. J. D. Arnal, Á. F. Arenas, and Á. L. Arbeloa, "Esophageal cancer: risk factors, screening and endoscopic treatment in Western and Eastern countries," World Journal of Gastroenterology, vol. 21, no. 26, pp. 7933-7943, 2015.

[5] X. Song, W. You, J. Zhu et al., "A genetic variant in miRNA-219-1 is associated with risk of esophageal squamous cell carcinoma in Chinese Kazakhs," Disease Markers, vol. 2015, Article ID 541531, 10 pages, 2015.

[6] B. Ye, C.-Y. Ji, Y. Zhao, W. Li, J. Feng, and X. Zhang, "Single nucleotide polymorphism at alcohol dehydrogenase-1B is associated with risk of esophageal squamous cell carcinoma," Cancer Cell International, vol. 14, no. 1, article 12, 2014.

[7] H. Meng, K. Wang, X. Chen et al., "MicroRNA-330-3p functions as an oncogene in human esophageal cancer by targeting programmed cell death 4," American Journal of Cancer Research, vol. 5, no. 3, pp. 1062-1075, 2015.

[8] K. A. Byrnes, P. Phatak, D. Mansour et al., "Overexpression of miR-199a-5p decreases esophageal cancer cell proliferation through repression of mitogen-activated protein kinase kinase kinase-11 (MAP3K11)," Oncotarget, vol. 7, no. 8, pp. 8756-8770, 2016.

[9] M. Zhang, E. Linghu, Q. Zhan et al., "Methylation of DACT2 accelerates esophageal cancer development by activating Wnt signaling," Oncotarget, vol. 7, no. 14, pp. 17957-17969, 2016.

[10] Y. Wang, X. Qin, J. Wu et al., "Association of promoter methylation of RUNX3 gene with the development of esophageal cancer: a meta analysis," PLoS ONE, vol. 9, no. 9, Article ID e107598, 2014.

[11] L. Gautier, L. Cope, B. M. Bolstad, and R. A. Irizarry, "Affyanalysis of Affymetrix GeneChip data at the probe level," Bioinformatics, vol. 20, no. 3, pp. 307-315, 2004.

[12] G. K. Smyth, "Limma: linear models for microarray data," in Bioinformatics and Computational Biology Solutions Using R and Bioconductor, pp. 397-420, Springer, 2005.

[13] Y. Benjamini and Y. Hochberg, "Controlling the false discovery rate: a practical and powerful approach to multiple testing," Journal of the Royal Statistical Society, Series B: Methodological, vol. 57, no. 1, pp. 289-300, 1995.

[14] M. Ashburner, C. A. Ball, J. A. Blake et al., "Gene ontology: tool for the unification of biology," Nature Genetics, vol. 25, no. 1, pp. 25-29, 2000.

[15] W. G. Alvord, J. Roayaei, R. Stephens et al., "The DAVID gene functional classification tool: a novel biological module-centric algorithm to functionally analyze large gene lists," Genome Biology, vol. 8, no. 9, article R183, 2007.

[16] M. Kanehisa, M. Araki, S. Goto et al., "KEGG for linking genomes to life and the environment," Nucleic Acids Research, vol. 36, supplement 1, pp. D480-D484, 2008.

[17] P. Shannon, A. Markiel, O. Ozier et al., "Cytoscape: a software environment for integrated models of biomolecular interaction networks," Genome Research, vol. 13, no. 11, pp. 2498-2504, 2003.

[18] A. Chatr-Aryamontri, B.-J. Breitkreutz, R. Oughtred et al., "The BioGRID interaction database: 2015 update," Nucleic Acids Research, vol. 43, no. 1, pp. D470-D478, 2015.

[19] T. D. Schmittgen and K. J. Livak, "Analyzing real-time PCR data by the comparative CT method," Nature Protocols, vol. 3, no. 6, pp. 1101-1108, 2008.

[20] T. Feng, F. Shao, Q. Wu et al., "miR-124 downregulation leads to breast cancer progression via LncRNA-MALAT1 regulation and CDK4/E2F1 signal activation," Oncotarget, vol. 7, no. 13, pp. 16205-16216, 2016.

[21] L. Yan, Y. Wang, Z.-Z. Wang et al., "Cell motility and spreading promoted by CEACAM6 through cyclin D1/CDK4 in human pancreatic carcinoma," Oncology Reports, vol. 35, no. 1, pp. 418426, 2016.

[22] H. Xiao, J. Zeng, H. Li et al., "MiR-1 downregulation correlates with poor survival in clear cell renal cell carcinoma where it interferes with cell cycle regulation and metastasis," Oncotarget, vol. 6, no. 15, pp. 13201-13215, 2015.

[23] J. Wang, S. Yu, L. Cui et al., "Role of SMC1A overexpression as a predictor of poor prognosis in late stage colorectal cancer," BMC Cancer, vol. 15, no. 1, article 90, 2015.

[24] T. Feng, D. Xu, C. Tu et al., "miR-124 inhibits cell proliferation in breast cancer through downregulation of CDK4," Tumor Biology, vol. 36, no. 8, pp. 5987-5997, 2015.

[25] Y. Zhang, Y. Wang, Y. Wei et al., "Molecular chaperone CCT3 supports proper mitotic progression and cell proliferation in hepatocellular carcinoma cells," Cancer Letters, vol. 372, no. 1, pp. 101-109, 2016.

[26] X. Cui, Z.-P. Hu, Z. Li, P.-J. Gao, and J.-Y. Zhu, "Overexpression of chaperonin containing TCP1, subunit 3 predicts poor prognosis in hepatocellular carcinoma," World Journal of Gastroenterology, vol. 21, no. 28, pp. 8588-8604, 2015.

[27] Z. Pénzváltó, A. Lánczky, J. Lénárt et al., “MEK1 is associated with carboplatin resistance and is a prognostic biomarker in epithelial ovarian cancer," BMC Cancer, vol. 14, no. 1, 2014.

[28] Y. Shi, X. Deng, Q. Zhan et al., "A prospective proteomic-based study for identifying potential biomarkers for the diagnosis of cholangiocarcinoma," Journal of Gastrointestinal Surgery, vol. 17, no. 9, pp. 1584-1591, 2013.

[29] J. Ma, X. Hou, M. Li et al., "Genome-wide methylation profiling reveals new biomarkers for prognosis prediction of glioblastoma," Journal of Cancer Research and Therapeutics, vol. 11, no. 6, pp. 212-215, 2015.

[30] H. Cohen, R. Ben-Hamo, M. Gidoni et al., "Shift in GATA3 functions, and GATA3 mutations, control progression and clinical presentation in breast cancer," Breast Cancer Research, vol. 16, no. 6, article 464, 2014.

[31] Y. Su, J. Wang, X. Zhang et al., "Targeting SIM2-s decreases glioma cell invasion through mesenchymal-epithelial transition," Journal of Cellular Biochemistry, vol. 115, no. 11, pp. 19001907, 2014.

[32] Y. Su, Q. He, L. Deng et al., "MiR-200a impairs glioma cell growth, migration, and invasion by targeting SIM2-s," NeuroReport, vol. 25, no. 1, pp. 12-17, 2014.

[33] W. H. Xiao, X. L. Qu, X. M. Li et al., "Identification of commonly dysregulated genes in colorectal cancer by integrating analysis of RNA-Seq data and qRT-PCR validation," Cancer Gene Ther$a p y$, vol. 22, no. 5, pp. 278-284, 2015. 
[34] B. Laffin, E. Wellberg, H.-I. Kwak et al., "Loss of singleminded$2 s$ in the mouse mammary gland induces an epithelialmesenchymal transition associated with up-regulation of slug and matrix metalloprotease 2," Molecular and Cellular Biology, vol. 28, no. 6, pp. 1936-1946, 2008.

[35] M. Komatsu and H. Sasaki, "DNA methylation is a key factor in understanding differentiation phenotype in esophageal squamous cell carcinoma," Epigenomics, vol. 6, no. 6, pp. 567-569, 2014.

[36] H.-I. Kwak, T. Gustafson, R. P. Metz, B. Laffin, P. Schedin, and W. W. Porter, "Inhibition of breast cancer growth and invasion by single-minded 2s," Carcinogenesis, vol. 28, no. 2, pp. 259-266, 2007.

[37] C. Rosenberg, E. Geelen, M. J. IJszenga et al., "Spectrum of genetic changes in gastro-esophageal cancer cell lines determined by an integrated molecular cytogenetic approach," Cancer Genetics and Cytogenetics, vol. 135, no. 1, pp. 35-41, 2002.

[38] B. Albrecht, M. Hausmann, H. Zitzelsberger et al., "Array-based comparative genomic hybridization for the detection of DNA sequence copy number changes in Barrett's adenocarcinoma," The Journal of Pathology, vol. 203, no. 3, pp. 780-788, 2004.

[39] S. Komatsu, I. Imoto, H. Tsuda et al., "Overexpression of SMYD2 relates to tumor cell proliferation and malignant outcome of esophageal squamous cell carcinoma," Carcinogenesis, vol. 30, no. 7, pp. 1139-1146, 2009.

[40] C. Fan, L. Chen, Q. Huang et al., "Overexpression of major CDKN3 transcripts is associated with poor survival in lung adenocarcinoma," British Journal of Cancer, vol. 113, no. 12, pp. 1735-1743, 2015.

[41] T. Li, H. Xue, Y. Guo, and K. Guo, "CDKN3 is an independent prognostic factor and promotes ovarian carcinoma cell proliferation in ovarian cancer," Oncology Reports, vol. 31, no. 4, pp. 1825-1831, 2014.

[42] J. Li, Z. Chen, L. Tian et al., "LncRNA profile study reveals a three-lncRNA signature associated with the survival of patients with oesophageal squamous cell carcinoma," Gut, vol. 63, no. 11, pp. 1700-1710, 2014.

[43] K. Chen, Y. Li, Y. Dai et al., "Characterization of tumor suppressive function of cornulin in esophageal squamous cell carcinoma," PLoS ONE, vol. 8, no. 7, Article ID e68838, 2013.

[44] A. Long, V. Giroux, K. A. Whelan et al., "WNT10A promotes an invasive and self-renewing phenotype in esophageal squamous cell carcinoma," Carcinogenesis, vol. 36, no. 5, pp. 598-606, 2015.

[45] T. Shimokuni, K. Tanimoto, K. Hiyama et al., "Chemosensitivity prediction in esophageal squamous cell carcinoma: novel marker genes and efficacy-prediction formulae using their expression data," International Journal of Oncology, vol. 28, no. 5, pp. 1153-1162, 2006.

[46] B. P. Mello, E. F. Abrantes, C. H. Torres et al., "No-match ORESTES explored as tumor markers," Nucleic Acids Research, vol. 37, no. 8, pp. 2607-2617, 2009. 


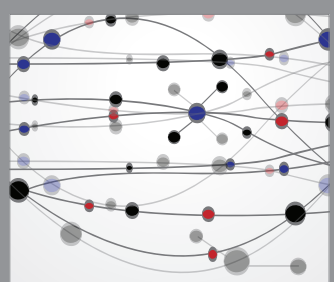

The Scientific World Journal
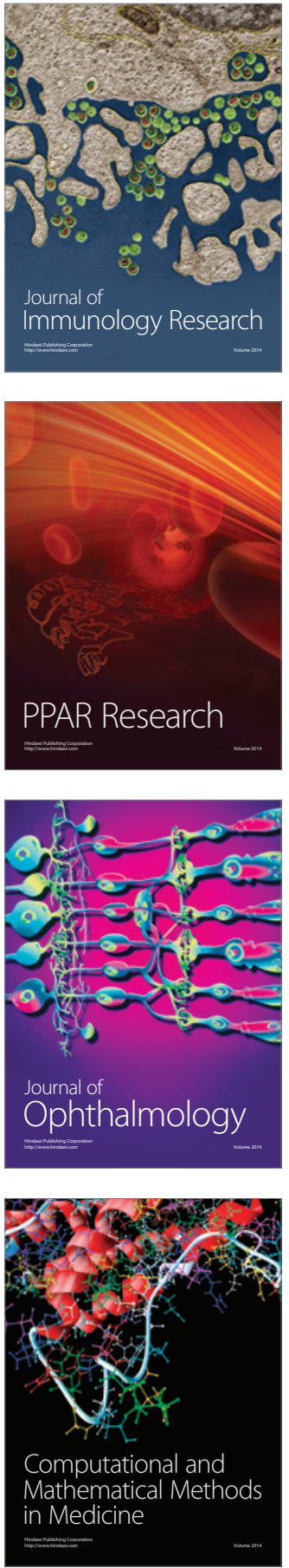

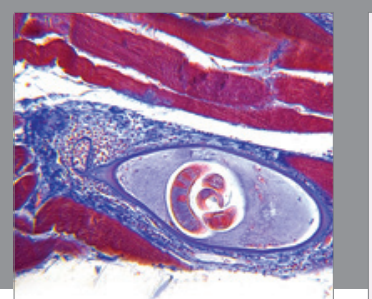

Gastroenterology Research and Practice

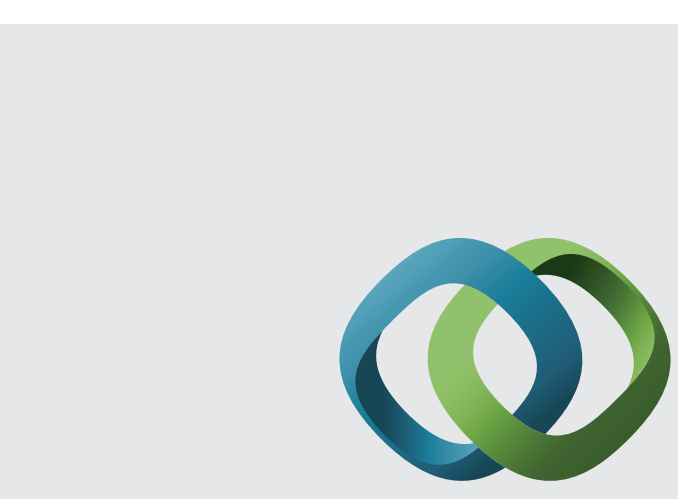

\section{Hindawi}

Submit your manuscripts at

http://www.hindawi.com
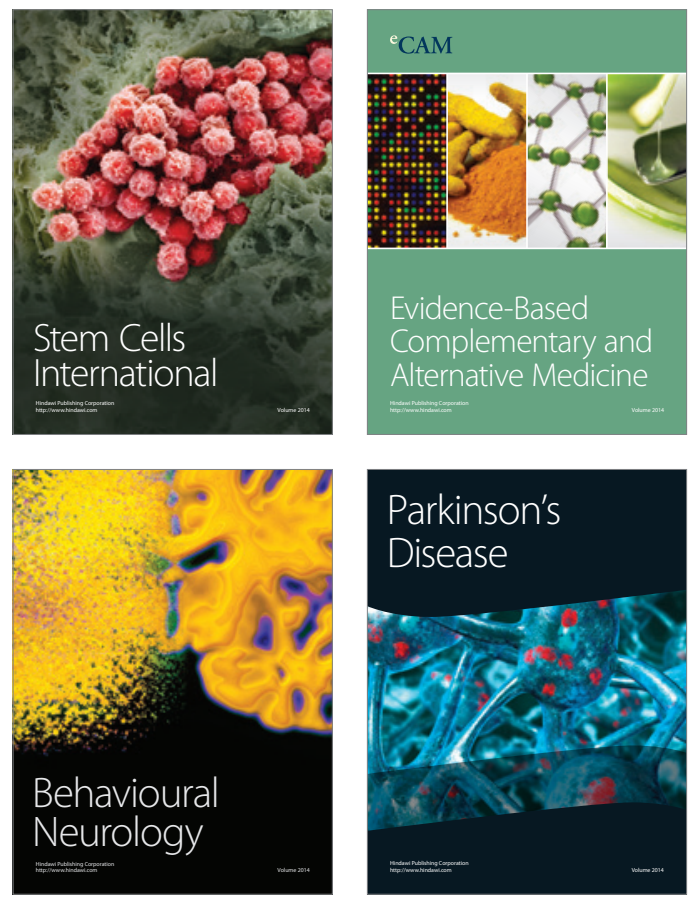
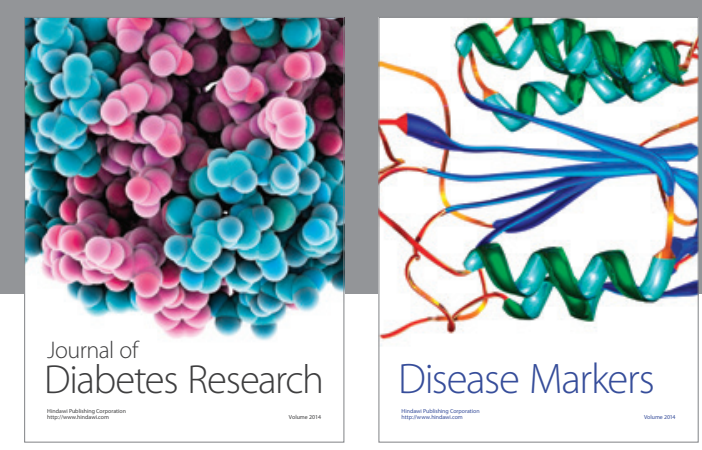

Disease Markers
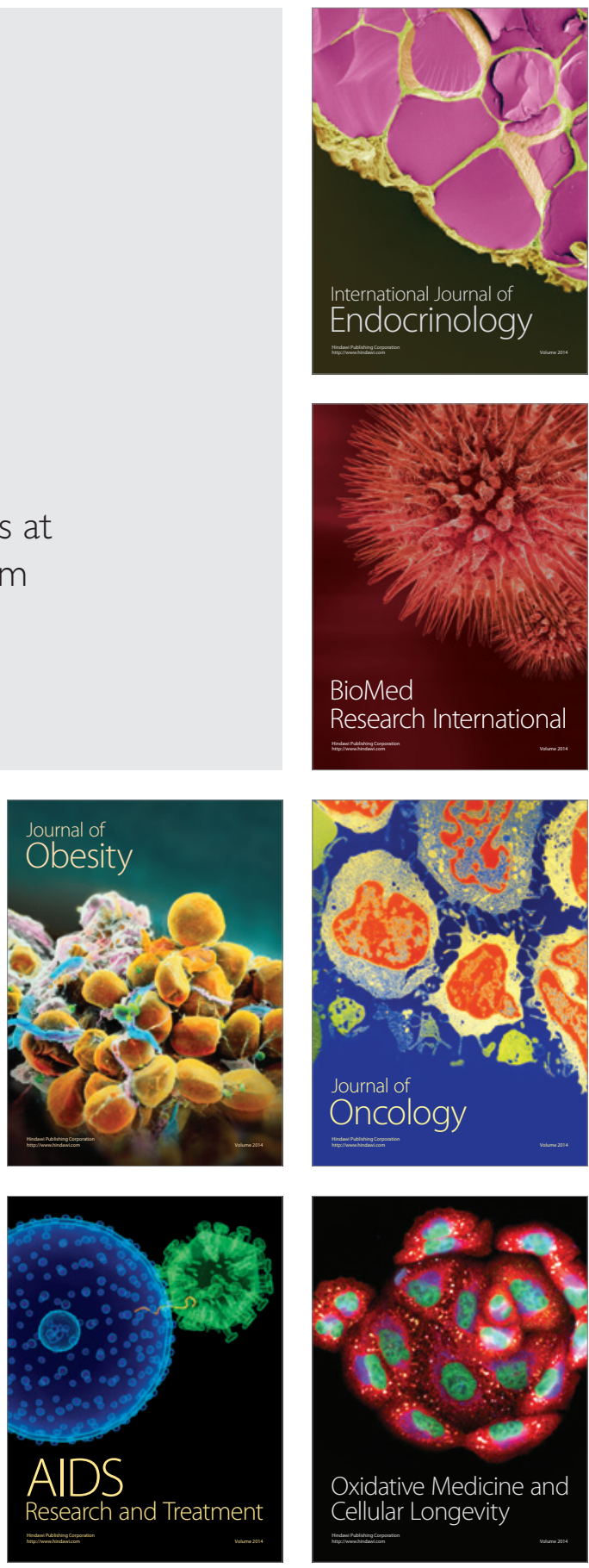